\title{
Understanding the ACM THRI Review Process
}

\author{
ODEST CHADWICKE JENKINS, University of Michigan \\ SELMA ŠABANOVIĆ, Indiana University Bloomington
}

\begin{abstract}
ACM Reference format:
Odest Chadwicke Jenkins and Selma Šabanović. 2018. Understanding the ACM THRI Review Process. ACM Trans. Hum.-Robot Interact. 7, 3, Article 18 (December 2018), 2 pages.

https://doi.org/10.1145/3289154
\end{abstract}

We are delighted to conclude our successful first year of $A C M$ THRI with an outstanding issue of articles representing a diverse range of thought in contemporary Human-Robot Interaction (HRI). The first contribution from our new Mechanical section, from Che et al., explores haptics for usable interaction with robotic assistants. This article presents a wearable haptic device for bi-directional human-robot communication in applications involving robust person-following by autonomous mobile robots. From our Computational section, Nikolaidis et al. investigate collaborative manipulation between robots and human partners through their introduction of a formal computational model for planning using verbal and non-verbal cues. The Behaviorial section features two contributions in social robotics. The first, by Leyzberg et al., explores social robotic tutors in the educational setting for English language learning through studying the effects and gains of adaptive personalization algorithms. Rosenthal-von der Pütten and Bock consider the acceptance and adoption of robots by human users through a new measure of self-efficacy uniquely suited to evaluating human-robot systems.

The completion of our first volume of ACM THRI also offers the opportunity to reflect on the accomplishments and challenges of the journal. When we started as editors-in-chief, our goals were threefold: (i) increase the impact of HRI as a field, (ii) provide sustainability in the timeliness and quality in our reviewing, and (iii) cultivate both diversity and excellence in our contributions. With the guidance and support of the ACM and the HRI Steering Committee, we faced the growing pains of transitioning the fournal of Human-Robot Interaction into its next phase as ACM THRI. Only time will tell whether our aims for intellectual impact and diverse excellence come to fruition. However, this time is opportune for taking stock of our current reviewing practices with one volume under our belt.

In short, the review processes established for ACM THRI have been quite successful in the quality of our review decisions and feedback, although our timeliness can be improved. Quality in our review feedback has been the highest priority of ACM THRI. We do not see reviewing as strictly

\footnotetext{
Authors' addresses: O. C. Jenkins, Department of Electrical Engineering and Computer Science, Robotics Institute, University of Michigan, 2260 Hayward St., Ann Arbor, MI 48109; email: ocj@umich.edu; S. Šabanović, School of Informatics, Computing and Engineering, Indiana University Bloomington, 901 E. 10th Street, Bloomington, IN 47408; email: selmas@ indiana.edu.

Permission to make digital or hard copies of part or all of this work for personal or classroom use is granted without fee provided that copies are not made or distributed for profit or commercial advantage and that copies bear this notice and the full citation on the first page. Copyrights for third-party components of this work must be honored. For all other uses, contact the Owner/Author.

2018 Copyright is held by the owner/author(s).

2573-9522/2018/12-ART18

https://doi.org/10.1145/3289154
} 
quality control to threshold submissions into bins of publishable works and rejected manuscripts. Instead, our greater responsibility is to provide feedback that will enable authors to improve their work toward publication or, in some cases, find new and more viable problems.

The essence of a quality ACM THRI review is the phrasing of an argument in the assessment of a manuscript. Regardless of whether a submission is accepted for publication, returned for revision, or rejected from consideration, a sound and considerate argument is essential for the cultivation of new intellectual understanding. Precision in the "why" of a review decision is more important than "what" specific decision results. We strive as a journal to provide a clear rationale for each decision we issue. Towards this end, every submission to ACM THRI is discussed among our editorial board, which includes the senior associate editors of each section and the editors-in-chief. Submissions that go through full review are analyzed by three expert reviewers, an associate editor, and our editorial board to construct the best review feedback possible.

Toward sound argumentation in our review process, ACM THRI has also developed and deployed a more precise reviewer scorecard than standard review forms. The typical review form is commonly structured as a collection of text boxes with summary numeric score. Such review forms are not in direct alignment with elucidating a sound argument. Depending on the attentiveness of the reviewer, this purely open-ended style of review can exacerbate bias and uncertainty with an overemphasis on the numeric score.

The ACM THRI reviewer scorecard aims to provide greater precision by linking the review form with a flow chart of binary yes or no decisions. Each binary decision addresses an important aspect of the review with regard to the dimensions of clarity, validity, novelty, relevance, and reproducibility. Further, each binary decision has a direct implication for the recommended review decision and should be supported with a explanation in text. For example, if a reviewer considers a manuscript to not be novel, the reviewer should provide citations to at least three papers that subsume this work with a rationale. Such supporting rationale not only helps us make sound editorial decisions but also helps inform authors such that they can produce better work for the future. The end result of answering all of these questions implicitly provides an argument and a recommended review decision.

Finally, we cannot emphasize enough how much we appreciate and value timely contributions from everyone involved in the journal. ACM THRI would not be a success without the efforts, insight, and spirit of those who review. We also remain keenly aware that our reviewers and editors are also authors themselves, who put incredible amount of time and care in their work. As such, our goal for ACM THRI is to return quality feedback in as timely a manner as possible, ideally with reviews returned in 14 weeks. One of our largest challenges remains to inspire reviewers in our community to respond to requests and return their reviews to faciliate this goal. So, please be thoughtful and timely in your reviewing to help us foster a virtuous cycle of intellectual argumentation. And through this virtuous cycle, we will make sure your papers get the same care and treatment from reviewers as you would want as an author. 